\title{
On the uniform input-to-state stability of reaction-diffusion systems
}

\author{
Sergey Dashkovskiy and Andrii Mironchenko
}

\begin{abstract}
In the present paper we consider uniform inputto-state stability of reaction-diffusion equations and compare it with its finite dimensional counterpart without diffusion as a parameterized set of decoupled equations. The reactiondiffusion partial differential equation can be seen as their interconnection via diffusion. We prove, that for linear reactiondiffusion systems and certain classes of nonlinear equations the UISS property for corresponding systems without diffusion implies, that the UISS property holds also for the system with diffusion.
\end{abstract}

Keywords: reaction-diffusion equations, input-to-state stability.

\section{INTRODUCTION}

In this paper we consider a distributed parameter system in form of parabolic partial differential equations (PDE's) and compare it with a continuum set of decoupled ordinary differential equations (ODE's). The system in form of a parabolic PDE can be seen as an interconnection of the set of the ODE's parameterized by a continuous spatial parameter via diffusion term. In both cases we consider systems with (the same) inputs and study stability properties. Our aim is to investigate the relation between stability properties of both kinds of systems. Since we consider systems with inputs we use the notion of input-to-state stability (ISS) that was introduced by E. Sontag [16] in 1989. This notion and related concepts (such as IOS, ISDS, LISS and other) have become a widely used framework for stability analysis of nonlinear control systems. This notion is very useful to investigate large interconnections of nonlinear systems. In particular, an interconnection of arbitrary number $n \in \mathbb{N}$ of ISS systems can be studied with help of small-gain conditions and related Lyapunov theory, see [4], [5], [10].

In the literature the notion of ISS was used for different kinds of systems including systems of ODE's [17], [8], [1] hybrid, switched and impulsive systems [6], [18], [3], systems with time delays [14], [7], as well as discrete time systems: [9], [13]. In the last years a big attention is devoted to boundary stabilization of PDE's [11], that confirms the importance of stability of distributed parameter systems.

In this paper we deal with time-varying systems, therefore we use the notion of uniform ISS (UISS), developed for this class of systems, see [19]. We are going to apply UISS notion

This research is funded by the German Research Foundation (DFG) as a part of Collaborative Research Centre 637 "Autonomous Cooperating Logistic Processes - A Paradigm Shift and its Limitations".

S. Dashkovskiy is with Department of Mathematics and Computer Science, University of Bremen, Bibliothekstraße 1, 28359 Bremen, Germany dsn@math. uni-bremen. de

A. Mironchenko is with Department of Mathematics and Computer Science, University of Bremen, Bibliothekstraße 1, 28359 Bremen, Germany andmiremath.uni-bremen.de to PDE's and to present some results, concerning uniform input-to-state stability of reaction-diffusion equations, under Neumann (no flux) boundary conditions.

We will prove, that for linear PDE systems, and some subclasses of nonlinear reaction-diffusion equations the investigation of the UISS property can be reduced to the exploration of the corresponding system without diffusion.

The paper has the following structure: in the next section the necessary notions are introduced. In the Section III the results are stated and proved, afterwards the simulation, illustrating one of the theorems is provided. Section V concludes the paper.

\section{PRELIMINARIES}

Let $|\cdot|$ denote Euclidean norm in $\mathbb{R}^{n}$ and $M$ be a subset in $\mathbb{R}^{p}$. Denote $\mathbb{R}_{\geq t_{0}}:=\left[t_{0},+\infty\right)$.

Since we are going to consider only classical solutions of parabolic equations we need the following function spaces:

$$
\begin{array}{r}
C\left(M, \mathbb{R}^{n}\right):=\left\{f: M \mapsto \mathbb{R}^{n} \mid\right. \\
\quad f \text { is continuous and bounded on } M\}, \\
C^{2,1}\left(M \times\left[t_{0}, T\right], \mathbb{R}^{n}\right):=\left\{s: M \times\left[t_{0}, T\right] \mapsto \mathbb{R}^{n} \mid\right. \\
\left.s_{x}, s_{x x}, s_{t} \text { are continuous and bounded on } M \times\left[t_{0}, T\right]\right\},
\end{array}
$$

where $t_{0}<T \in \mathbb{R}$. The norm on $C\left(M, \mathbb{R}^{n}\right)$ is defined by $\|f\|_{C\left(M, \mathbb{R}^{n}\right)}:=\sup _{x \in M}|f(x)|$. In case $M$ is compact sup can be replaced with max in this definition.

Let $G$ be a bounded domain in $\mathbb{R}^{p}$ with a smooth boundary $\partial G$, and $\Delta$ denote the Laplacian operator on $G$. We study reaction-diffusion system with Neumann (no flux) boundary conditions:

$$
\left\{\begin{array}{l}
\frac{\partial s(x, t)}{\partial t}=c^{2} \Delta s+f(s, x, t, u(x, t)), x \in G, t>t_{0}, \\
s\left(x, t_{0}\right)=\phi_{0}(x), x \in G, \\
\left.\frac{\partial s}{\partial n}\right|_{\partial G \times \mathbb{R}_{\geq t_{0}}=0}=0
\end{array}\right.
$$

where $c$ is a constant, $u: G \times \mathbb{R}_{\geq t_{0}} \rightarrow \mathbb{R}^{m}$ is a continuous input signal and $f: \mathbb{R}^{n} \times G \times \mathbb{R}_{\geq t_{0}} \times \mathbb{R}^{m} \rightarrow \mathbb{R}^{n}$ satisfies certain regularity properties such that there exists a unique classical solution $s$ of the problem (1) such that $s \in C(\bar{G} \times$ $\left.\left[t_{0}, T\right], \mathbb{R}^{n}\right) \cap C^{2,1}\left(G \times\left[t_{0}, T\right], \mathbb{R}^{n}\right)$, and has bounded first and second order derivatives with respect to $x$ in $\bar{G}$. For the appropriate regularity conditions we refer, e.g., to [12].

We consider the asymptotic behavior of the solutions $s$ for a problem (1), thereby we need their existence and uniqueness for all $T>t_{0}$.

In addition to (1) we consider the corresponding system without diffusion of the form:

$$
\left\{\begin{array}{l}
\frac{\partial s(x, t)}{\partial t}=f(s, x, t, u(x, t)), x \in G, t>t_{0}, \\
s(x, 0)=\phi_{0}(x), x \in G .
\end{array}\right.
$$


This equation can be understood as a system of ordinary differential equations, parameterized by spatial parameter $x \in G$. We assume, that function $f$ is at least Lipschitz continuous on $s$, uniformly in $t$ and $u$ for all $x \in G$, and continuous on $x$ to guarantee the existence and uniqueness of solutions to problem (2).

Definition 1: For the stability analysis the following classes of functions are useful:

$$
\begin{aligned}
& \mathcal{K}:=\left\{\gamma: \mathbb{R}_{+} \rightarrow \mathbb{R}_{+} \mid \gamma \text { is continuous, } \gamma(0)=0\right. \\
& \mathcal{K}_{\infty}:=\{\gamma \in \mathcal{K} \mid \gamma \text { is unbounded }\} \\
& \text { and strictly increasing } \\
& \mathcal{L} \quad:=\left\{\gamma: \mathbb{R}_{+} \rightarrow \mathbb{R}_{+} \mid \gamma\right. \text { is continuous and strictly } \\
& \text { decreasing with } \left.\lim _{t \rightarrow \infty} \gamma(t)=0\right\} \\
& \mathcal{K} \mathcal{L}:=\left\{\beta: \mathbb{R}_{+} \times \mathbb{R}_{+} \rightarrow \mathbb{R}_{+} \mid \beta\right. \text { is continuous, } \\
& \beta(\cdot, t) \in \mathcal{K}, \beta(r, \cdot) \in \mathcal{L}, \forall t, r \geq 0\}
\end{aligned}
$$

Let $\phi\left(t, t_{0}, \phi_{0}, u\right)$ be the solution of the system (1) (or (2)) corresponding to the initial conditions $s\left(\cdot, t_{0}\right)=\phi_{0}(\cdot)$ and external input $u$.

We assume, that $f(0, x, t, 0) \equiv 0 \forall(x, t) \in G \times \mathbb{R}_{\geq t_{0}}$ so that the origin is an equilibrium point for both systems (1) and (2).

For the sake of brevity we use in the paper the following notation: $C(G):=C\left(G, \mathbb{R}^{n}\right), \quad C\left(\mathbb{R}_{\geq t_{0}}\right):=$ $C\left(\mathbb{R}_{\geq t_{0}}, \mathbb{R}^{m}\right), C\left(G \times \mathbb{R}_{\geq t_{0}}\right):=C\left(G \times \mathbb{R}_{\geq t_{0}}, \mathbb{R}^{m}\right)$.

Since we work with time-varying systems, we use the following stability notions from [19] for systems (1) and (2):

Definition 2: The distributed parameter system (1) (or (2)) is uniformly globally asymptotically stable at zero (0-UGAS) on $G$, if $\exists \beta \in \mathcal{K} \mathcal{L}$, such that the following inequality

$$
\left\|\phi\left(t, t_{0}, \phi_{0}, 0\right)\right\|_{C(G)} \leq \beta\left(\left\|\phi_{0}\right\|_{C(G)}, t-t_{0}\right)
$$

holds $\forall \phi_{0} \in C(G), \forall t \geq t_{0}$.

Definition 3: The distributed parameter system (1) (or (2)) is uniformly input-to-state stable (UISS) on $G$, if $\exists \beta \in \mathcal{K} \mathcal{L}$ and $\gamma \in \mathcal{K}$, such that the inequality

$$
\begin{aligned}
& \left\|\phi\left(t, t_{0}, \phi_{0}, u\right)\right\|_{C(G)} \\
& \quad \leq \beta\left(\left\|\phi_{0}\right\|_{C(G)}, t-t_{0}\right)+\gamma\left(\|u\|_{C\left(G \times \mathbb{R}_{\geq t_{0}}\right)}\right)
\end{aligned}
$$

holds $\forall \phi_{0} \in C(G), \forall t \geq t_{0}$ and $\forall u \in C\left(G \times \mathbb{R}_{\geq t_{0}}\right)$.

For any fixed $x_{0} \in G$ we consider also a system of ODEs, corresponding to (2)

$$
\left\{\begin{array}{l}
\frac{d s\left(x_{0}, t\right)}{\partial t}=f\left(s, x_{0}, t, u\left(x_{0}, t\right)\right), t \geq t_{0}, \\
s\left(x_{0}, t_{0}\right)=\phi_{0}\left(x_{0}\right) .
\end{array}\right.
$$

The solution of this system we denote by $\phi_{x_{0}}\left(t, t_{0}, \phi_{0}\left(x_{0}\right), u\left(x_{0}, t\right)\right)$.

Definition 4: The system (5) is uniformly input-to-state stable, if $\exists \beta_{x_{0}} \in \mathcal{K} \mathcal{L}$ and $\gamma_{x_{0}} \in \mathcal{K}$, such that the inequality

$$
\begin{aligned}
& \left|\phi_{x_{0}}\left(t, t_{0}, \phi_{0}\left(x_{0}\right), u\left(x_{0}, t\right)\right)\right| \\
& \leq \beta_{x_{0}}\left(\left|\phi_{0}\left(x_{0}\right)\right|, t-t_{0}\right)+\gamma_{x_{0}}\left(\left\|u\left(x_{0}, \cdot\right)\right\|_{C\left(\mathbb{R}_{\geq t_{0}}\right)}\right)
\end{aligned}
$$

holds $\forall \phi_{0}\left(x_{0}\right) \in \mathbb{R}^{n}, \forall t \geq t_{0}$ and $\forall u\left(x_{0}, \cdot\right) \in C\left(\mathbb{R}_{\geq t_{0}}\right)$.

Note, that the region $G$ in definitions of 0-UGAS and UISS must be open only for system (1). For systems (2) and (5) $G$ can be an arbitrary set in $\mathbb{R}^{p}$.

The aim of this paper is to prove, that for certain classes of reaction-diffusion equations the UISS property for the system
(2) implies the UISS property for the system (1). Also we prove, that UISS of system (2) on $K$, where $K$ is compact, is equivalent to UISS of ODE system (5) in every point $x_{0} \in K$, provided that values of functions $\beta_{x_{0}}(r, s)$ and $\gamma_{x_{0}}(r)$ depend continuously on the parameter $x_{0}$ in $K$.

In other words we are going to derive the following set of implications (here "UISS" means UISS on $G$ ):

(5) is UISS $\forall x_{0} \in G \Leftrightarrow(2)$ is UISS $\Rightarrow$ (1) is UISS.

Thus, for such classes of systems the question about uniform input-to-state stability of a reaction-diffusion system can be resolved to the question of uniform input-to-state stability of the ODE system, for which theory is already welldeveloped. In the next section we do it for linear reactiondiffusion systems, and for a certain class of nonlinear reaction-diffusion systems.

\section{RESULTS}

Firstly we prove a technical lemma, that we could not find in the literature, but need for the proof of Theorem 1.

Lemma 1: Let $\left(R_{X}, \rho_{X}\right)$ and $\left(R_{Y}, \rho_{Y}\right)$ be metric spaces, and $X \subset R_{X}$ be a compact set, $Y \subset R_{Y}$. Assume, that $f: X \times Y \mapsto \mathbb{R}$ is continuous on $X \times Y$. Then function $g(y)=\max _{x \in X} f(x, y)$ is continuous on $Y$.

Proof: Denote by $X_{y}:=\arg \max _{x \in X} f(x, y)$ - the set of values $x \in X$, on which a function $f(\cdot, y)$ takes its maximum.

At first we are going to prove auxiliary statement, namely $\forall \omega>0 \exists \delta>0$, such that

$$
\forall y \in Y: \rho_{Y}\left(y, y_{0}\right)<\delta \Rightarrow X_{y} \subset U_{\omega}\left(X_{y_{0}}\right)=\bigcup_{x \in X_{y_{0}}} U_{\omega}(x),
$$

where $U_{\omega}(x)$ is a ball with centre in $x$ and radius $\omega$.

Assume, that this statement does not hold. Then there exists $\omega_{0}>0$ and sequences $\left\{\delta_{n}\right\}_{n=1}^{n=\infty}, \lim \delta_{n}=0$, $\left\{y_{n}\right\}_{n=1}^{n=\infty} \subset Y, \rho_{Y}\left(y_{n}, y_{0}\right)<\delta_{n}$ and $\left\{x_{n}\right\}_{n=1}^{n \rightarrow \infty} \begin{gathered}n=\infty \\ n=1\end{gathered}$, where $x_{n} \in X_{y_{n}} \backslash U_{\omega_{0}}\left(X_{y_{0}}\right)$.

By construction $\lim _{n \rightarrow \infty} y_{n}=y_{0} . X$ is compact, therefore $\left\{x_{n}\right\}_{n=1}^{n=\infty}$ is bounded, and from Bolzano-Weierstrass theorem it follows, that a convergent subsequence $\left\{x_{n_{k}}\right\}_{k=1}^{k=\infty} \subset$ $\left\{x_{n}\right\}_{n=1}^{n=\infty}$ exists. Let $\lim _{k \rightarrow \infty}\left(x_{n_{k}}, y_{n_{k}}\right)=\left(x^{*}, y_{0}\right) \in X \times Y$.

If $x^{*} \in X_{y_{0}}$, then some elements of $\left\{x_{n_{k}}\right\}_{k=1}^{k=\infty}$ belong to $U_{\omega_{0}}\left(x^{*}\right) \subset U_{\omega_{0}}\left(X_{y_{0}}\right)$, and we have a contradiction.

Let $x^{*} \notin X_{y_{0}}$. Then $f\left(x^{*}, y_{0}\right)<f\left(x_{0}, y_{0}\right)$ for some $x_{0} \in$ $X_{y_{0}}$, and therefore disjunct balls $U_{s}\left(\left(x^{*}, y_{0}\right)\right) \subset X \times Y$ and $U_{s}\left(\left(x_{0}, y_{0}\right)\right) \subset X \times Y$ for some $s>0$ exist, such that $\forall\left(x^{\prime}, y^{\prime}\right) \in U_{s}\left(\left(x^{*}, y_{0}\right)\right), \forall(x, y) \in U_{s}\left(\left(x_{0}, y_{0}\right)\right)$ it holds $f\left(x^{\prime}, y^{\prime}\right)<f(x, y)$. But $\forall s>0$ in $U_{s}\left(\left(x^{*}, y_{0}\right)\right)$ infinitely many elements of the sequence $\left(x_{n_{k}}, y_{n_{k}}\right)$ exist. Let one of them be $\left(x_{n_{k 1}}, y_{n_{k 1}}\right)$. We have, that function $f\left(\cdot, y_{n_{k 1}}\right)$ does not possess maximum at $x_{n_{k 1}}$, and therefore $x_{n_{k 1}} \notin X_{y_{n_{k 1}}}$, and we come to a contradiction. Our statement is proven.

Now we can prove the claim of the lemma. We have, that $\forall \omega>0 \exists \delta_{1}>0$, such that $\forall y \in Y$ with $\rho_{Y}\left(y, y_{0}\right)<\delta_{1}$ there exist $x_{1} \in X_{y}, x_{0} \in X_{y_{0}}$ with $\rho_{X}\left(x_{1}, x_{0}\right)<\omega$, and 
we have

$$
\begin{aligned}
& \left|g(y)-g\left(y_{0}\right)\right|=\left|\max _{x \in X} f(x, y)-\max _{x \in X} f\left(x, y_{0}\right)\right| \\
& =\left|f\left(x_{1}, y\right)-f\left(x_{0}, y_{0}\right)\right| \\
& \leq\left|f\left(x_{1}, y\right)-f\left(x_{1}, y_{0}\right)\right|+\left|f\left(x_{1}, y_{0}\right)-f\left(x_{0}, y_{0}\right)\right| .
\end{aligned}
$$

From the continuity of $f\left(x_{1}, \cdot\right)$ at the point $y_{0}$ we have, that $\forall \varepsilon_{2}>0 \exists \delta_{2}: \forall y \in Y: \rho_{Y}\left(y, y_{0}\right)<\delta_{2} \Rightarrow \mid f\left(x_{1}, y\right)-$ $f\left(x_{1}, y_{0}\right) \mid<\varepsilon_{2}$.

To estimate $\left|f\left(x_{1}, y_{0}\right)-f\left(x_{0}, y_{0}\right)\right|$ we use that $\forall y_{0} \in$ $Y$ function $f\left(\cdot, y_{0}\right)$ is a continuous function, defined on a compact set, and, according to Heine-Cantor theorem, it is uniformly continuous, that is $\forall \varepsilon_{1}>0 \exists \omega: \forall x_{1}, x_{0} \in X$ : $\rho_{X}\left(x_{1}, x_{0}\right)<\omega \Rightarrow\left|f\left(x_{1}, y_{0}\right)-f\left(x_{0}, y_{0}\right)\right|<\varepsilon_{1}$.

Taking $\delta=\min \left\{\delta_{1}, \delta_{2}\right\}$, and $\varepsilon=\varepsilon_{1}+\varepsilon_{2}$, we have, that $\forall \varepsilon>0 \exists \delta>0$ such that

$$
\forall y \in Y: \rho_{Y}\left(y, y_{0}\right)<\delta \Rightarrow\left|g(y)-g\left(y_{0}\right)\right|<\varepsilon .
$$

Thus, $g$ is continuous at $y_{0}$. Since $y_{0}$ was chosen arbitrarily, $g$ is continuous in all $Y$.

Also we need the following

Lemma 2: Let $M$ be a subset of $\mathbb{R}^{p}$, and system (2) be UISS on $M$. Then (2) is UISS on every $S, S \subset M$.

Proof: Let $\phi\left(t, t_{0}, \phi_{0}, u\right)$ be a solution of (2) on $M$ (thus, $\phi_{0}$ is defined on $M$ ) and $\phi^{r}\left(t, t_{0}, \phi_{0}^{r}, u\right), \phi_{0}^{r}$ and $u^{r}$ be restrictions of $\phi\left(t, t_{0}, \phi_{0}, u\right), \phi_{0}$ and $u$ to $S \subset M$, that is $\phi_{0}^{r}, u^{r}(\cdot, t) \in C(S), \phi_{0}^{r} \equiv \phi_{0}$ on $S, u^{r}(\cdot, t) \equiv u(\cdot, t)$ on $S$ and $\phi^{r}\left(t, t_{0}, \cdot, u\right)$ is a function from $C(S)$ into $C(S)$. We have:

$$
\begin{gathered}
\left\|\phi^{r}\left(t, t_{0}, \phi_{0}^{r}, u^{r}\right)\right\|_{C(S)} \leq\left\|\phi\left(t, t_{0}, \phi_{0}, u\right)\right\|_{C(M)} \\
\leq \beta\left(\left\|\phi_{0}\right\|_{C(M)}, t-t_{0}\right)+\gamma\left(\|u\|_{C\left(M \times \mathbb{R}_{\geq t_{0}}\right)}\right) .
\end{gathered}
$$

These estimates are valid $\forall t \geq t_{0}$, for all admissible functions $\phi_{0}$ and $u$. For all $\phi_{0}^{r}$ and $u^{r}$ there exist continuous functions $\phi_{0}$ and $u$, such that $\phi_{0}^{r}$ and $u^{r}$ are their restrictions to the set $S$ and moreover $\left\|\phi_{0}\right\|_{C(M)}=\left\|\phi_{0}^{r}\right\|_{C(S)}$, and $\|u\|_{C\left(M \times \mathbb{R}_{\geq t_{0}}\right)}=\left\|u^{r}\right\|_{C\left(S \times \mathbb{R}_{\geq t_{0}}\right)}$.

From the last estimates we obtain:

$$
\begin{gathered}
\left\|\phi^{r}\left(t, t_{0}, \phi_{0}^{r}, u^{r}\right)\right\|_{C(S)} \\
\leq \beta\left(\left\|\phi_{0}^{r}\right\|_{C(S)}, t-t_{0}\right)+\gamma\left(\left\|u^{r}\right\|_{C\left(S \times \mathbb{R}_{\geq t_{0}}\right)}\right),
\end{gathered}
$$

which holds $\forall t \geq t_{0}, \phi_{0}^{r} \in C(S), u^{r} \in C\left(S \times \mathbb{R}_{\geq t_{0}}\right)$. Hence we conclude that (2) is UISS on $S$.

Remark 3: Note, that for a system (1) the claim of this lemma is not true.

Now we are ready to prove, that UISS property for a system (2) on compact set $K \subset \mathbb{R}^{p}$ is equivalent to UISS property of a system (5) in every point $x_{0} \in K$.

Theorem 1: Consider problem (2) with $x \in K, K \subset \mathbb{R}^{p}$ is compact. Let the values of functions $\beta_{x_{0}}$ and $\gamma_{x_{0}}$ from Definition 4 depend on parameter $x_{0}$ continuously on $K$. Then system (2) is UISS on $K$ if and only if system (5) is UISS for all $x_{0} \in K$.

Proof: Let system (5) be UISS $\forall x_{0} \in K$, i.e., for each $x_{0} \in K$ there exist $\beta_{x_{0}} \in \mathcal{K} \mathcal{L}$ and $\gamma_{x_{0}} \in \mathcal{K}$, such that inequality (6) holds $\forall \phi_{0}\left(x_{0}\right) \in \mathbb{R}^{n}, \forall t \geq t_{0}, \forall u\left(x_{0}, \cdot\right) \in$ $C\left(\mathbb{R}_{\geq t_{0}}\right)$.

We introduce functions $\beta$ and $\gamma$ by the following:

$$
\beta(r, t):=\max _{x_{0} \in K} \beta_{x_{0}}(r, t), \gamma(r):=\max _{x_{0} \in K} \gamma_{x_{0}}(r),
$$

where $r, t \in \mathbb{R}_{\geq 0}$.

By this definition it holds $\forall x_{0} \in K, \forall t \geq t_{0}$, that

$$
\begin{aligned}
& \beta_{x_{0}}\left(\left|\phi_{0}\left(x_{0}\right)\right|, t-t_{0}\right) \leq \beta\left(\left\|\phi_{0}\right\|_{C(K)}, t-t_{0}\right), \\
& \gamma_{x_{0}}\left(\left\|u\left(x_{0}, \cdot\right)\right\|_{C\left(\mathbb{R}_{\geq t_{0}}\right)}\right) \leq \gamma\left(\|u\|_{C\left(K \times \mathbb{R}_{\geq t_{0}}\right)}\right) .
\end{aligned}
$$

Functions $\beta$ and $\gamma$ exist and are continuous functions on their domains of definition by the Lemma 1 . The strict monotonicity can be shown as follows. Let $0 \leq a<b$ and let the maximum values $\gamma(a)$ and $\gamma(b)$ be attained in $x_{a}$ and $x_{b}$ respectively. We have

$$
\gamma(b)=\gamma_{x_{b}}(b) \geq \gamma_{x_{a}}(b)>\gamma_{x_{a}}(a)=\gamma(a) .
$$

This shows that $\gamma$ is a $\mathcal{K}$-function. Similarly one can show that $\beta \in \mathcal{K} \mathcal{L}$.

From inequality (6), using (7) and estimates (8), (9), we have:

$$
\begin{aligned}
& \left|\phi_{x_{0}}\left(t, t_{0}, \phi_{0}\left(x_{0}\right), u\left(x_{0}, t\right)\right)\right| \leq \\
& \beta\left(\left\|\phi_{0}\right\|_{C(K)}, t-t_{0}\right)+\gamma\left(\|u\|_{C\left(K \times \mathbb{R}_{\geq t_{0}}\right)}\right) .
\end{aligned}
$$

This inequality holds $\forall x_{0} \in K, \forall t \geq t_{0}$. Taking maximum over all $x_{0} \in K$, and using, that

$$
\max _{x_{0} \in K}\left|\phi_{x_{0}}\left(t, t_{0}, \phi_{0}\left(x_{0}\right), u\left(x_{0}, t\right)\right)\right|=\left\|\phi\left(t, t_{0}, \phi_{0}, u\right)\right\|_{C(K)}
$$

we obtain, that system (2) is UISS on $K$ with respect to the norm $\|\cdot\|_{C}$. So, from the UISS of system (5) in every point $x_{0} \in K$ it follows UISS of the system (2) on $K$.

To prove the theorem in the other direction, it is enough to apply Lemma 2 for $S=\left\{x_{0}\right\}$ for all $x_{0} \in K$.

Note, that formulas (7) provide us with the gains for the system (2), if we know the gains for system (5). Of course, if $\beta_{x_{0}}$ and $\gamma_{x_{0}}$ do not depend on parameter $x_{0}$ continuously, maximums may not exist, and (7) cannot be applied.

Next we consider several special cases.

\section{A. Linear systems}

In this subsection we consider the autonomous systems, therefore UISS is equivalent to the usual ISS property.

Let the nonhomogeneous part of (1) be linear with respect to $s$

$$
\left\{\begin{array}{l}
\frac{\partial s(x, t)}{\partial t}=c^{2} \Delta s+R s+f(x, u(x, t)), x \in G, t>t_{0}, \\
s\left(x, t_{0}\right)=\phi_{0}(x), x \in G, \\
\left.\frac{\partial s}{\partial n}\right|_{\partial G \times \mathbb{R}_{\geq t_{0}}}=0,
\end{array}\right.
$$

where $R \in \mathbb{R}^{n \times n}$ and $f$ is continuous with respect to both arguments on $\bar{G} \times \mathbb{R}^{m}$ and $\phi_{0} \in C(G)$. The corresponding system without diffusion is:

$$
\left\{\begin{array}{l}
\frac{\partial \tilde{s}(x, t)}{\partial t}=R \tilde{s}+f(x, u(x, t)), x \in G, t>t_{0}, \\
\tilde{s}\left(x, t_{0}\right)=\phi_{0}(x), x \in G .
\end{array}\right.
$$

We assume, that $f(x, 0)=0$, thus, $s(x, t) \equiv 0$ is the only equilibrium solution of the systems (11) and (12). 
Theorem 2: (11) is UISS on $G \Leftrightarrow(12)$ is UISS on $G$.

Proof: We begin with sufficiency. Let system (11) be UISS, and therefore, 0-UGAS. We consider the linear ODE system with parameter $x_{0}$, corresponding to the system (12):

$$
\left\{\begin{array}{l}
\frac{d \tilde{s}\left(x_{0}, t\right)}{d t}=R \tilde{s}+f\left(x_{0}, u\left(x_{0}, t\right)\right), \\
\tilde{s}\left(x_{0}, t_{0}\right)=\phi_{0}\left(x_{0}\right) .
\end{array}\right.
$$

Consider (11) and (12) with $u \equiv 0$ (thus, $f(x, u) \equiv 0$ ). Fix arbitrary $\phi_{0}\left(x_{0}\right) \in \mathbb{R}^{n}$ in the initial condition of (13). And let $s^{*}$ be the corresponding solution of (13). But $s^{*}$ solves also (12) and (11) with the same initial condition $\phi_{0}(x) \equiv$ $\phi_{0}\left(x_{0}\right)$. By the 0 -UGAS property of (11) we have

$$
\left|s^{*}(t)\right| \leq \beta\left(\left\|\phi_{0}\right\|_{C(G)}, t-t_{0}\right)=\beta\left(\left|\phi_{0}\left(x_{0}\right)\right|, t-t_{0}\right), t \geq 0,
$$

i.e., (13) is 0-UGAS. Hence matrix $R$ is Hurwitz.

Functions $\beta_{x_{0}}$ and $\gamma_{x_{0}}$ for linear system (13) can be computed analytically, and are continuous with respect to $x_{0} \in \bar{G}$. Using Theorem 1 , we see, that (12) is UISS on $\bar{G}$, and, by Lemma 2, on $G$.

Now we proceed with necessity. Let (12) be UISS on $G$, then the matrix $R$ is Hurwitz. Let $J$ be Jordan normal form of the matrix $R$. Then invertible matrix $U \in \mathbb{C}^{n \times n}$ exists, such that $R=U J U^{-1}$. Substituting it into the system (11), and introducing variables $y=U^{-1} s, g(x, u(x, t))=$ $U^{-1} f(x, u(x, t))$ and $\psi(x)=U^{-1} \phi_{0}(x)$, we obtain:

$$
\left\{\begin{array}{l}
\frac{\partial y(x, t)}{\partial t}=c^{2} \Delta y+J y+g(x, u(x, t)), x \in G, t>t_{0}, \\
y\left(x, t_{0}\right)=\psi(x), x \in G, \\
\left.\frac{\partial y}{\partial n}\right|_{\partial G \times \mathbb{R}_{\geq t_{0}}=0 .}
\end{array}\right.
$$

This system is linear, therefore its solution can be taken as $y(x, t)=y_{1}(x, t)+y_{2}(x, t)$, where $y_{1}(x, t)$ is the solution of the homogeneous system

$$
\left\{\begin{array}{l}
\frac{\partial y_{1}(x, t)}{\partial t}=c^{2} \Delta y_{1}+J y_{1}, x \in G, t>t_{0} \\
y_{1}\left(x, t_{0}\right)=\psi(x), x \in G \\
\left.\frac{\partial y_{1}}{\partial n}\right|_{\partial G \times \mathbb{R}_{\geq t_{0}}}=0
\end{array}\right.
$$

and $y_{2}(x, t)$ is the solution of the nonhomogeneous system with $\psi \equiv 0$ :

$$
\left\{\begin{array}{l}
\frac{\partial y_{2}(x, t)}{\partial t}=c^{2} \Delta y_{2}+J y_{2}+g(x, u(x, t)) \\
y_{2}\left(x, t_{0}\right)=0, x \in G \\
\left.\frac{\partial y_{2}}{\partial n}\right|_{\partial G \times \mathbb{R}_{\geq t_{0}}}=0 .
\end{array}\right.
$$

We claim, that $\exists \beta \in \mathcal{K} \mathcal{L}$ and $\exists \gamma \in \mathcal{K}$, such that $\left\|y_{1}(\cdot, t)\right\|_{C(G)} \leq \beta\left(\left\|\phi_{0}\right\|_{C(G)}, t-t_{0}\right)$, and $\left\|y_{2}(\cdot, t)\right\|_{C(G)} \leq$ $\gamma\left(\|u\|_{C\left(G \times \mathbb{R}_{\geq t_{0}}\right)}\right)$.

We prove only second claim, the other case is analogous. The last equation in (16) has the form

$$
\frac{\partial y_{2}^{n}(x, t)}{\partial t}=c^{2} \Delta y_{2}^{n}+\lambda_{n} y_{2}^{n}+g_{n}(x, u(x, t)),
$$

where $\lambda_{n}=\alpha_{n}+\beta_{n} i, \alpha_{n}<0$, and $y_{2}=\left(y_{2}^{1}, y_{2}^{2}, \ldots, y_{2}^{n}\right)^{T}$. By the substitution $y_{2}^{n}:=e^{\beta_{n} i t} h_{n}$, in this equation we obtain

$$
\frac{\partial h_{n}(x, t)}{\partial t}=c^{2} \Delta h_{n}+\alpha_{n} h_{n}+\tilde{g}_{n}(x, u(x, t))
$$

where $\tilde{g}_{n}(x, u(x, t))=e^{-\beta_{n} i t} g_{n}(x, u(x, t))$. Note, that $h\left(x, t_{0}\right) \equiv y_{2}\left(x, t_{0}\right) \equiv 0$.

For all input signals $u$ with $\|u\|_{C\left(G \times \mathbb{R}_{\geq t_{0}}\right)}<\delta$ by the continuity of $g$ it follows that there exist

$$
g^{-}=\inf _{G \times \mathbb{R}_{\geq t_{0}}} \tilde{g}_{n}(x, u(x, t)), g^{+}=\sup _{G \times \mathbb{R}_{\geq t_{0}}} \tilde{g}_{n}(x, u(x, t)) .
$$

Due to linearity solutions of the last equation are bounded from below and above by the solutions for the same equation with $\tilde{g}_{n}(x, u(x, t))$ replaced with $g^{-}$and $g^{+}$respectively, which are bounded since $\alpha_{n}<0$. This shows that $\left|y_{2}^{n}\right|$ is bounded by some constant that depends on $\delta$.

By the same arguments we can prove that $y_{2}^{i}$ is bounded from below and above for $i=1, \ldots, n$. In particular it follows that $\left\|y_{2}(\cdot, t)\right\|_{C(G)} \leq \gamma\left(\|u\|_{C\left(G \times \mathbb{R}_{\geq t_{0}}\right)}\right)$ for some $\gamma \in \mathcal{K}$.

Finally, using the triangle inequality

$$
\|y(\cdot, t)\|_{C(G)} \leq\left\|y_{1}(\cdot, t)\right\|_{C(G)}+\left\|y_{2}(\cdot, t)\right\|_{C(G)},
$$

we finish the proof.

1) Positive systems: For the applications a special case, when $s(x, t) \geq 0$ (note, that this condition adds restrictions on the set of admissible controls) is very important, because such physical values, as absolute temperature, concentration of a chemical substance, population density cannot be negative. In this subsection we assume, that all solutions $s$ and $\tilde{s}$ of (11) and (12) respectively are nonnegative.

In this case we can prove more, namely that functions $\beta$ and $\gamma$ coincide for both (11) and (12) systems in case of UISS property, if we change the function norm in the definition of UISS.

Recall that $\forall t \geq t_{0} L_{1}$-norm on $G$ with respect to $x$ of the function $\phi\left(t, t_{0}, s_{0}, u\right)$ is defined as follows:

$$
\left\|\phi\left(t, t_{0}, s_{0}, u\right)\right\|_{L_{1}}=\int_{G}|s(x, t)| d G .
$$

Subtracting $\tilde{s}$ from $s$ it follows from (11) and (12) that

$$
\left\{\begin{array}{l}
\frac{\partial(s-\tilde{s})}{\partial t}=c^{2} \Delta s+R(s-\tilde{s}) \\
(s-\tilde{s})\left(x, t_{0}\right)=0, x \in G \\
\left.\frac{\partial s}{\partial n}\right|_{\partial G \times \mathbb{R}_{\geq t_{0}}=0}
\end{array}\right.
$$

We define: $A(t)=\int_{G} s(x, t) d G, \tilde{A}(t)=\int_{G} \tilde{s}(x, t) d G$. By the Gauß divergence theorem and Neumann boundary conditions we get $\int_{G} \Delta s d G=\int_{\partial G} \frac{\partial s}{\partial n} d S=0$, hence from (17) we have:

$$
\left\{\begin{array}{l}
\frac{\partial(A-\tilde{A})}{\partial t}=R(A-\tilde{A}), \\
(A-\tilde{A})\left(x, t_{0}\right)=0, x \in G .
\end{array}\right.
$$

It follows that $A-\tilde{A} \equiv 0$, and for nonnegative solutions we have:

$$
\left\|\phi\left(t, t_{0}, s_{0}, u\right)\right\|_{L_{1}}=\left\|\tilde{\phi}\left(t, t_{0}, s_{0}, u\right)\right\|_{L_{1}}
$$

So, $L_{1}$ norm of the solutions and, consequently, gains (if we consider UISS property with respect to the $L_{1}$-norm instead of $C$-norm) of the systems (11) and (12) are equal. 


\section{B. Nonlinear monotone systems}

Let us introduce the partial order $\leq$ on $\mathbb{R}^{n}$ by the relation

$$
x \leq y \quad \Leftrightarrow \quad x_{i} \leq y_{i}, i=1, \ldots, n .
$$

We use the following definition (compare with definition of monotone control systems in [2]):

Definition 5: We call system (1) monotone with respect to initial data, if for all initial conditions $s_{1}\left(x, t_{0}\right), s_{2}\left(x, t_{0}\right) \in$ $C(G)$, such that $s_{1}\left(x, t_{0}\right) \geq s_{2}\left(x, t_{0}\right), \forall x \in G$ it holds $s_{1}(x, t) \geq s_{2}(x, t), \forall t>t_{0}, \forall x \in G, \forall u \in C(G \times$ $\mathbb{R}_{\geq t_{0}}$ ), where $s_{i}(x, t), i=1,2$ are the solutions of (1), corresponding to some input $u$.

In this section we analyse system (1) for the case, when $f=f(s, t, u(t))$ :

$$
\left\{\begin{array}{l}
\frac{\partial s(x, t)}{\partial t}=c^{2} \Delta s+f(s, t, u(t)), x \in G, t>t_{0}, \\
s\left(x, t_{0}\right)=\phi_{0}(x), x \in G, \\
\left.\frac{\partial s}{\partial n}\right|_{\partial G \times \mathbb{R}_{\geq t_{0}}}=0 .
\end{array}\right.
$$

We assume, that $f(0, t, 0)=0 \forall t \geq t_{0}$.

Theorem 3: Let system (19) be monotone with respect to initial data. If the corresponding to (19) system without diffusion is UISS on $G$, then (19) is also UISS on $G$.

Proof: For arbitrary $\phi_{0} \in C(G)$ we define constant vectors $\phi_{+}, \phi_{-}$by:

$$
\phi_{+}^{i}=\sup _{x \in G} \phi_{0}^{i}(x), \quad \phi_{-}^{i}=\inf _{x \in G} \phi_{0}^{i}(x), i=1, \ldots, n .
$$

Consider problem (19) with $\phi_{0}(x)=\phi_{+}, \forall x \in G$ and $\phi_{0}(x)=\phi_{-}, \forall x \in G$ (with corresponding solutions $s_{+}(x, t)$ and $s_{-}(x, t)$ respectively).

Using, that $f$ and $u$ do not depend on $x$, we see, as in the proof of the Theorem 2, that $s_{+}(x, t) \equiv s_{+}^{*}(t) \forall x \in G$, where $s_{+}^{*}$ is a solution of the problem

$$
\left\{\begin{array}{l}
\frac{\partial s_{+}^{*}(t)}{\partial t}=f\left(s_{+}^{*}(t), t, u(t)\right), t>t_{0}, \\
s_{+}^{*}\left(t_{0}\right)=\phi_{+} .
\end{array}\right.
$$

Thus $\left\|s_{+}(\cdot, t)\right\|_{C(G)}=\left|s_{+}^{*}(t)\right|$. Similarly, $\left\|s_{-}(\cdot, t)\right\|_{C(G)}=\left|s_{-}^{*}(t)\right|$, where $s_{-}^{*}$ is a solution of (21) with $\phi_{-}$instead of $\phi_{+}$.

But we know, that the non-diffusional system is UISS, therefore $\exists \beta_{*} \in \mathcal{K} \mathcal{L}, \gamma_{*} \in \mathcal{K}$, such that $\forall \phi_{-}, \phi_{+} \in \mathbb{R}^{n}$, $\forall u \in C\left(\mathbb{R}_{\geq t_{0}}\right), \forall t \geq t_{0}$ it holds

$$
\begin{aligned}
\left\|s_{-}(\cdot, t)\right\|_{C(G)} & =\left|s_{-}^{*}(t)\right| \\
& \leq \beta_{*}\left(\left|\phi_{-}\right|, t-t_{0}\right)+\gamma_{*}\left(\|u\|_{C\left(\mathbb{R}_{\geq t_{0}}\right)}\right) . \\
\left\|s_{+}(\cdot, t)\right\|_{C(G)} & =\left|s_{+}^{*}(t)\right| \\
& \leq \beta_{*}\left(\left|\phi_{+}\right|, t-t_{0}\right)+\gamma_{*}\left(\|u\|_{C\left(\mathbb{R}_{\geq t_{0}}\right)}\right) .
\end{aligned}
$$

From definitions (20) it follows, that

$$
\phi_{-} \leq \phi_{0}(x) \leq \phi_{+}, \forall x \in G .
$$

Now, from the assumption of monotonicity of (19) with respect to initial data, we know, that for any admissible $\phi_{0}$ for the corresponding solution $s(x, t)$ of the problem (19) it holds:

$$
s_{-}(\cdot, t) \leq s(\cdot, t) \leq s_{+}(\cdot, t), \forall t \geq t_{0} .
$$

Now, if $a \leq x \leq b, a, b, x \in \mathbb{R}^{n}$ then

$$
|x|=\sqrt{\sum_{i=1}^{n} x_{i}^{2}} \leq \sqrt{\sum_{i=1}^{n}\left(a_{i}^{2}+b_{i}^{2}\right)} \leq|a|+|b| .
$$

Thus,

$$
\|s(\cdot, t)\|_{C(G)} \leq\left\|s_{-}(\cdot, t)\right\|_{C(G)}+\left\|s_{+}(\cdot, t)\right\|_{C(G)} .
$$

The following simple inequalities will be useful for us:

$$
\left|\phi_{-}\right| \leq \sqrt{n}\left\|\phi_{0}\right\|_{C(G)}, \quad\left|\phi_{+}\right| \leq \sqrt{n}\left\|\phi_{0}\right\|_{C(G)} .
$$

Using these estimates, we have:

$$
\begin{gathered}
\|s(\cdot, t)\|_{C(G)} \leq \beta_{*}\left(\left|\phi_{-}\right|, t-t_{0}\right)+\beta_{*}\left(\left|\phi_{+}\right|, t-t_{0}\right) \\
+\gamma_{*}\left(\|u\|_{C\left(\mathbb{R}_{\geq t_{0}}\right)}\right)+\gamma_{*}\left(\|u\|_{C\left(\mathbb{R}_{\geq t_{0}}\right)}\right) \\
\leq 2 \beta_{*}\left(\sqrt{n}\left\|\phi_{0}\right\|_{C(G)}, t-t_{0}\right)+2 \gamma_{*}\left(\|u\|_{C\left(\mathbb{R}_{\geq t_{0}}\right)}\right) .
\end{gathered}
$$

Taking $\beta(r, t):=2 \beta_{*}(\sqrt{n} r, t), x \in \mathbb{R}_{\geq 0}, t \in \mathbb{R}_{\geq t_{0}}$, and $\gamma:=2 \gamma_{*}$, we obtain, that for solution $s(x, t)$ of the problem (19) it holds

$$
\|s(\cdot, t)\|_{C(G)} \leq \beta\left(\left\|\phi_{0}\right\|_{C(G)}, t-t_{0}\right)+\gamma\left(\|u\|_{C\left(\mathbb{R}_{\geq t_{0}}\right)}\right)
$$

for all initial functions $\phi_{0} \in C(G)$ and external inputs $u \in$ $C\left(\mathbb{R}_{\geq t_{0}}\right)$.

\section{EXAMPLE}

In this section we demonstrate the applicability of the theorem 3 on the example.

Take $G=[-1,1]^{2}$ and consider the following equation $(s(x, t) \in \mathbb{R})$ on $G$ :

$$
\left\{\begin{array}{l}
\frac{\partial s(x, t)}{\partial t}=\Delta s-s^{3}(x, t)+u(t), x \in G, t>0, \\
s(x, 0)=\phi_{0}(x), x \in G, \\
\left.\frac{\partial s}{\partial n}\right|_{\partial G \times \mathbb{R}_{\geq 0}}=0,
\end{array}\right.
$$

For $v(t) \equiv 0$ the monotonicity of the system follows from the corollary 3.5 in [15]. In presence of input $v(t)$ it will be monotone with respect to initial data.

We solve it numerically on time span $[0,5]$ for the initial value

$$
\phi_{0}(x)=\left\{\begin{array}{l}
1,|x|<0.4 \\
0, \text { else }
\end{array}\right.
$$

and for the input $v(t)=2 \sin (10 t)+4$.

The solution $u$ for $t=0.3$ is presented in Figure 1: 


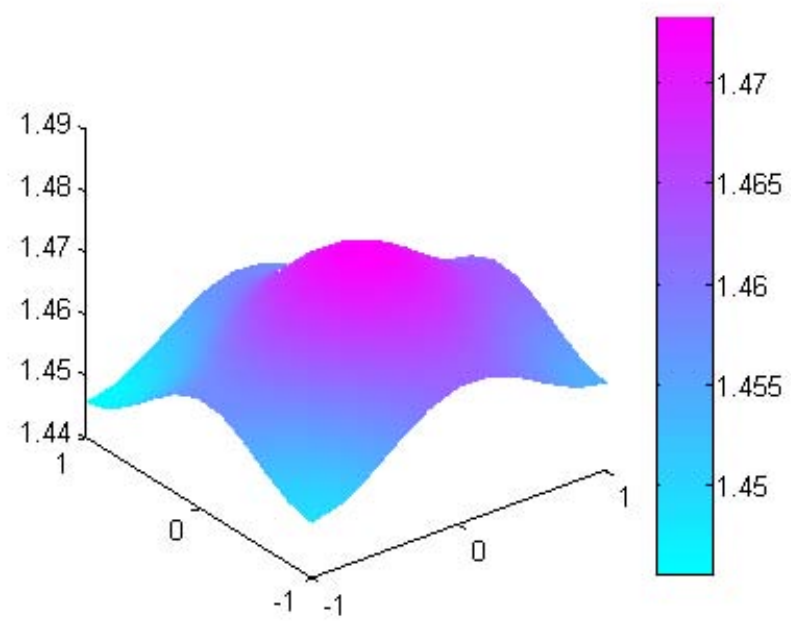

Fig. 1. Graph of $u(\cdot, 0.3)$

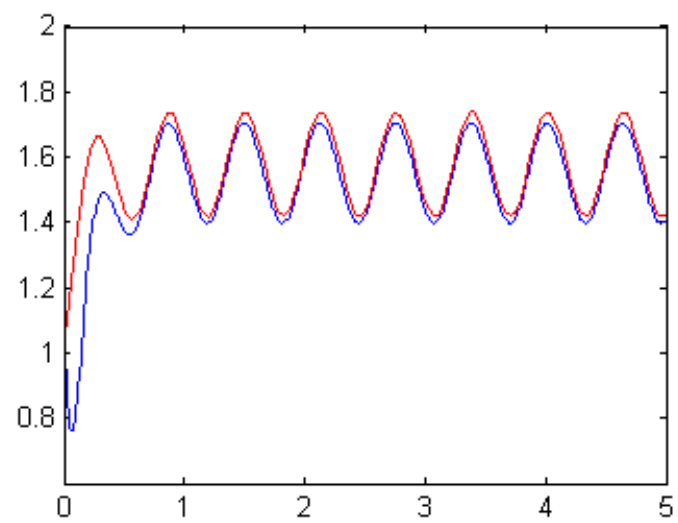

Fig. 2. Graph of $u_{+}$(above) and graph of $\|u(x, \cdot)\|_{C(G)}$

In Figure 2 the graphs of $\|u(x, \cdot)\|_{C(G)}$ (below) and of $u_{+}$ (above), where $u_{+}$is a solution of a problem $\dot{u}=-u^{3}(t)+$ $v(t), u(0)=\left\|\phi_{0}\right\|_{C(G)}$, are presented.

From 2 one sees, that $\|u(x, \cdot)\|_{C(G)}$ is bounded from above by $u_{+}$, in agreement with the claim of the theorem 3 .

The simulation was done, using discretization of time interval with the implicit Euler scheme (with step size $h=$ 0.01). The obtained after discretization elliptic equation was solved, using standard MATLAB solver for nonlinear elliptic equations (pdenonlin).

\section{Conclusions}

In the paper we have proved, that for linear reactiondiffusion equations and a certain subclass of nonlinear systems the analysis of uniform input-to-state stability can be resolved to the analysis of the uniform input-to-state stability of the corresponding ODE system. The result was illustrated with the help of simulation example. The question, whether this implication holds or not in general case, remains for the future investigations.

\section{REFERENCES}

[1] David Angeli. An almost global notion of input-to-state stability. IEEE Trans. Automat. Control, 49(6):866-874, 2004.

[2] David Angeli and Eduardo D. Sontag. Monotone control systems. IEEE Trans. Automat. Control, 48(10):1684-1698, 2003. New directions on nonlinear control.

[3] C. Cai and Andrew R. Teel. Characterizations of input-to-state stability for hybrid systems. Systems Control Lett., 58:47-53, 2009.

[4] Sergey Dashkovskiy, Björn S. Rüffer, and Fabian R. Wirth. An ISS small gain theorem for general networks. Math. Control Signals Systems, 19(2):93-122, 2007.

[5] Sergey N. Dashkovskiy, Björn S. Rüffer, and Fabian R. Wirth. Small Gain Theorems for Large Scale Systems and Construction of ISS Lyapunov Functions. SIAM Journal on Control and Optimization, 48(6):4089-4118, 2010.

[6] João P. Hespanha, Daniel Liberzon, and Andrew R. Teel. Lyapunov conditions for input-to-state stability of impulsive systems. Automatica J. IFAC, 44(11):2735-2744, 2008.

[7] H. Ito, P. Pepe, and Zhong-Ping Jiang. A small-gain condition for integral input-to-state stability of interconnected retarded nonlinear systems. In Proceedings of the 47th IEEE Conference on Decision and Control, 2008, pages $19-24$, dec. 2008.

[8] Zhong-Ping Jiang, Iven M. Y. Mareels, and Yuan Wang. A Lyapunov formulation of the nonlinear small-gain theorem for interconnected ISS systems. Automatica J. IFAC, 32(8):1211-1215, 1996.

[9] Zhong-Ping Jiang and Yuan Wang. Input-to-state stability for discretetime nonlinear systems. Automatica J. IFAC, 37(6):857-869, 2001.

[10] Iasson Karafyllis and Zhong-Ping Jiang. A vector smallgain theorem for general nonlinear control systems, 2009. http://arxiv.org/pdf/0904.0755.

[11] Miroslav Krstic. Systematization of approaches to adaptive boundary stabilization of PDEs. Internat. J. Robust Nonlinear Control, 16(16):801-818, 2006

[12] O. A. Ladyženskaja, V. A. Solonnikov, and N. N. Uralceva. Linear and quasilinear equations of parabolic type. Translated from the Russian by S. Smith. Translations of Mathematical Monographs, Vol. 23. American Mathematical Society, Providence, Rhode Island, 1967.

[13] Bin Liu and David J. Hill. Input-to-state stability for discrete timedelay systems via the Razumikhin technique. Systems Control Lett., 58(8):567-575, 2009

[14] P. Pepe, I. Karafyllis, and Z.-P. Jiang. On the Liapunov-Krasovskii methodology for the ISS of systems described by coupled delay differential and difference equations. Automatica J. IFAC, 44(9):22662273, 2008.

[15] Hal L. Smith. Monotone dynamical systems, volume 41 of Mathematical Surveys and Monographs. American Mathematical Society, Providence, RI, 1995. An introduction to the theory of competitive and cooperative systems.

[16] Eduardo D. Sontag. Smooth stabilization implies coprime factorization. IEEE Trans. Automat. Control, 34(4):435-443, 1989.

[17] Eduardo D. Sontag and Yuan Wang. New characterizations of inputto-state stability. IEEE Trans. Automat. Control, 41(9):1283-1294, 1996

[18] L. Vu, D. Chatterjee, and D. Liberzon. Input-to-state stability of switched systems and switching adaptive control. Automatica J. IFAC, 43(4):639-646, 2007

[19] Y. Wang Y. Lin and D. Cheng. On nonuniform and semi-uniform input-to-state stability for time-varying systems. In IFAC World Congress, Prague, 2005. 